DOI: 10.17234/SRAZ.65.52

\title{
Le varietà di lingua presenti nei sillabi dei corsi di lingua italiana nelle università in Polonia
}

\author{
Marta Kaliska \\ Università di Varsavia \\ m.kaliska@uw.edu.pl
}

Nel presente articolo si esporranno i risultati di uno studio di caso sul contenuto dei sillabi dei corsi di lingua italiana utilizzati nelle dieci più grandi università pubbliche in Polonia. La ricerca è stata svolta negli anni 2017-2018 e mirava all'esame delle nozioni di varietà presenti in tali documenti di pianificazione didattica.

Parole chiave: istruzione universitaria, sillabo, curricolo, didattica dell'italiano LS, varietà di lingua

\section{Introduzione}

Dal punto di vista di uno straniero, l'italiano contemporaneo non è una lingua omogenea nata da un modello nazionale storicamente diffuso in tutto il territorio dell'Italia odierna, bensì è una lingua impregnata di varietà, sia di quelle tipiche per ogni lingua come varietà diafasiche, diastratiche, diamesiche, che di quelle diatopiche risultanti dal contatto della lingua nazionale con i dialetti locali. L'apprendimento dell'italiano richiede un adeguato approfondimento della problematica legata alla variazione linguistica. Pertanto, abbiamo intrapreso uno studio di caso incentrato sul contenuto dei programmi d'insegnamento rispetto alla presenza delle nozioni di varietà, intendendo verificare in primo luogo, se tali componenti tematiche vengono prese in considerazione nella pianificazione didattica, in secondo, quali loro sottocategorie vengono messe in rilievo e quali invece trascurate o del tutto omesse.

\section{La didattica delle lingue nel contesto universitario}

La didattica delle lingue straniere al livello accademico deve essere mirata alla realizzazione degli obiettivi specifici degli studenti che da un lato si correlano con le necessità professionali, dall'altro con particolari contesti comunicativi della loro attività socioculturale.

A partire dall'implementazione delle riforme del Processo di Bologna, la didattica delle lingue nel contesto universitario si basa sui documenti, come 
Quadro comune europeo di riferimento per le lingue: apprendimento, insegnamento, valutazione (2002), Universities and language policy in Europe. Reference document (2001) e su altre raccomandazioni stese da parte del Consiglio d'Europa, della Commissione Europea e del Consiglio Europeo per le Lingue (Beacco, Byram 2007; Mezzadri 2016). In seguito alla loro approvazione le università hanno introdotto gli stessi descrittori dei livelli di competenza, sistemi di valutazione, contenuti tematici e obiettivi di insegnamento. Il QCER è divenuto un documento di riferimento essenziale, essendo focalizzato su tutte le aree della didattica: dalla metodologia e la definizione della competenza comunicativa alla valutazione del percorso di apprendimento. Nel 2018 è stata pubblicata la versione aggiornata del Quadro, il Companion Volume with New Descriptors in cui si ampliano i descrittori concernenti fenomeni più attuali, come l'abilità di mediazione e si espone il livello Pre-A1. Il documento costituisce una fonte essenziale per la pianificazione dei corsi in tutte le lingue.

\section{La programmazione didattica nel contesto universitario}

L'organizzazione del processo didattico rappresenta un compito complesso le cui tappe principali includono: la determinazione degli obiettivi generali nel curriculo degli studi ${ }^{1}$, la specificazione dei contenuti tematici e delle questioni organizzative nei sillabi. In quanto all'insegnamento delle lingue straniere nelle università in Polonia, le modalità della pianificazione dei corsi di lingua sono state adattate alle linee guida delle riforme europee che mirano all'internalizzazione degli atenei attraverso i programmi di scambio come ad esempio Erasmus, progetti di ricerca, nonché la promozione del plurilinguismo (Berlin Communiqué 2003).

La didattica delle lingue si dirama in tre principali categorie organizzative: la prima si riferisce ai lettorati, ossia ai corsi di lingue straniere di profilo generale dedicati a tutti gli studenti universitari di varie discipline che per conseguire la laurea breve o quella magistrale devono frequentare un dato numero di ore (120 ore nel caso degli studenti della triennale) e raggiungere il livello B2 o C1 secondo il QCER (Cisowska 2012). I lettorati di solito vengono coordinati dalle scuole o centri di lingue universitari che sono responsabili della preparazione di un'ampia offerta dei corsi e, spesso, anche degli esami certificatori alla fine di un percorso didattico.

La seconda categoria concerne la didattica delle lingue nell'ambito degli studi linguistico-filologici rivolti agli studenti i quali intendono occuparsi professionalmente delle lingue, per lo più in qualità di traduttori o docenti. Il curriculum viene incentrato non solo sulla stessa lingua, ma anche sui contenuti specialistici legati al tipo della specializzazione prescelta da parte degli

1 Con il termine curriculum o curriculo si intende un programma complessivo degli studi in cui si stabiliscono gli obiettivi educativi generali, gli obiettivi specifici da raggiungere in un dato periodo di tempo, i contenuti distribuiti per gli anni accademici che di seguito vengono esposti più dettagliatamente nei sillabi dei corsi (Cini 2011). 
studenti: traduzione o didattica. Lo stesso insegnamento delle lingue risulta più approfondito rispetto ai lettorati, il numero di ore ammonta a 240 o 300 l'anno e gli studenti devono raggiungere i livelli più alti: C1 (laurea triennale) e C2 (laurea magistrale).

Il terzo filone organizzativo, abbastanza recente, è mirato allo sviluppo integrato delle competenze linguistiche e professionali degli studenti di varie discipline, come economia, psicologia, risorse umane, ecc. È Content-Based Teaching che consiste nella preparazione non di un corso di lingua specialistica nel senso stretto, bensì di un corso tematico svolto in una lingua straniera, prevalentemente in inglese (Gajewska 2008). Per poter accedere a tali corsi gli studenti devono già dimostrare la conoscenza della lingua straniera al livello intermedio (preferibilmente B2) o avanzato $(\mathrm{C} 1, \mathrm{C} 2)$. L'obiettivo di tali corsi è anche quello di attirare gli studenti stranieri in un dato ateneo.

In quanto alle modalità di pianificazione dei corsi di lingua, il documento in cui si descrive il contenuto del corso è il sillabo che, nel caso dei lettorati rappresenta un programma a sé stante, invece nel caso dei corsi linguisticofilologici o corsi specialistici, rientra nel curricolo degli studi.

Il modello del sillabo introdotto nelle università polacche è un documento uniformato elaborato in base alle disposizioni dei Quadri Nazionali delle Qualifiche (Krajowe Ramy Kwalifikacji) approvati nel 2010 da parte del Ministero della Scienza e dell'Istruzione Superiore (Kraśniewski 2009). Con il termine sillabo si intende un documento di programmazione concernente un dato corso universitario in cui si espongono tutte le relative informazioni. La definizione utilizzata in questo studio corrisponde alla proposta di Cini secondo la quale, il sillabo serve "alla descrizione dei contenuti specifici da insegnare in un corso" (Cini 2011: 145). Va precisato che, parlando di questi contenuti specifici, ci si riferisce alle modalità organizzative (corso in classe, a distanza), agli obiettivi didattici, alla metodologia dell'insegnamento (tecniche e strategie prescelte), alla tematica (strutture grammaticali, lessico, ecc.), alle modalità di valutazione o ai risultati di apprendimento.

Tutti i sillabi sottoposti alla seguente analisi rappresentano il modello unificato i cui contenuti differenziano soltanto al livello tematico, ma non a quello strutturale.

\section{L'analisi del corpus dei sillabi}

Il corpus di ricerca è composto da 100 documenti rilevati nelle dieci più grandi università pubbliche in Polonia individuate in base a due criteri: il primo concerne il numero totale degli studenti secondo i dati statistici pubblicati nel 2017 da parte dell'Istituto Centrale di Statistica in Polonia (GUS 2017: 25) e il secondo - la presenza dei dipartimenti di italianistica. Di conseguenza, si sono selezionati i dieci più grandi atenei dove è possibile studiare la filologia italiana e sono: Università di Varsavia, Università 'Adam Mickiewicz' di Poznań, Università di Łódź, Università di Danzica, Università di Breslavia, Università 'Mikołaj 
Kopernik' di Toruń, Università della Slesia, Università 'Maria Curie-Skłodowska' di Lublino, Università di Warmia e Mazuria e Università di Stettino (Kaliska 2018). Tutti i sillabi del corpus sono stati codificati con i numeri di riferimento per una loro appropriata classificazione.

I documenti del corpus sono stati suddivisi in modo traversale in due categorie principali: a seconda della tipologia del corso: i lettorati e i corsi di stampo linguistico-filologico PNJW (in polacco Praktyczna nauka języka włoskiego, ossia 'l'insegnamento pratico della lingua italiana') e a seconda del livello di competenza: i documenti del livello intermedio A2/B1 e quelli più avanzati B2/ C1. Dall'analisi sono stati esclusi sia i sillabi al livello A1, visto che l'introduzione delle varietà richiede maggiori abilità linguistiche da parte degli apprendenti, che i sillabi dei corsi di linguaggi specialistici. Il corpus include soltanto i sillabi dei corsi generali utilizzati negli anni accademici 2016/17 e 2017/18. Si è deciso di escludere dalla ricerca i corsi specialistici organizzati sia dalle facoltà linguisticofilologiche che dalle scuole di lingua universitarie, perché tali corsi rappresentano unità tematiche sensu stricto e il loro sillabo è mirato soprattutto allo sviluppo delle competenze linguistiche specifiche per un dato contesto professionale, mentre il nostro obiettivo è stata la verifica della presenza di diverse varietà di lingua di ambito diafasico, diastratico e diatopico. Pertanto, si sono sottoposti all'esame i documenti di profilo linguistico generale che rappresentano un percorso didattico più ampio non limitato soltanto all'acquisizione di competenze specifiche.

Va aggiunto che nel corpus si sono trovati tre documenti di programmazione che inquadrano un insegnamento complessivo dal livello A1 a C1, il cui contenuto è stato esaminato soltanto rispetto ai livelli soprannominati, prescindendo dal livello più basso A1.

Nella tabella numero 1 sono stati illustrati i dati quantitativi riguardo al corpus.

\begin{tabular}{|c|c|c|c|}
\hline $\begin{array}{l}\text { Tipologia del corso } \\
\text { Livello di competenza }\end{array}$ & PNJW & Lettorati & Numero tot. \\
\hline Sillabi complessivi da A1 a C1 & 2 & 1 & 3 \\
\hline Sillabi A2/B1 & 29 & 24 & 53 \\
\hline Sillabi B2/C1 & 30 & 14 & 44 \\
\hline In totale & 61 & 39 & $\mathrm{~N}=100$ \\
\hline
\end{tabular}

Tab. 1. La suddivisione dei sillabi rilevati riguardo alla tipologia del corso e al livello di competenza.

Il nostro presupposto iniziale è stato quello di verificare se nell'insegnamento dell'italiano si prendono in considerazione le varietà dell'italiano che illustrano la sua ricca realtà sociolinguistica e quali varietà incluse nei programmi prevalgono. La metodologia di ricerca si è basata sull'analisi del contenuto rispetto alla presenza di dati elementi linguistici (Krippendorff 2004). Il primo 
passo è consistito nella identificazione delle nozioni di varietà: se una nozione era presente nel sillabo esaminato la si registrava come presenza (1), se invece risultava assente, si registrava assenza (0). Nel secondo passo, si è proceduti alla suddivisione delle nozioni in categorie concettuali più generali, tuttavia mantenendo la forma originale delle nozioni rilevate nell'ambito della categoria.

Si è deciso di sottoporre all'esame le varietà di lingua sistemate secondo tre assi di variazione (Berruto 1993): quello diatopico, diafasico e diastratico, prescindendo dalla variazione diamesica, visto che la ricerca mirava soprattutto all'analisi della rappresentazione sociolinguistica della lingua italiana. Con il termine 'varietà' si è intesa "un'entità discreta riconoscibile per gli addensamenti in un continuum al cui interno si riproduce un continuo" (Berruto 1993: 16).

Dunque, l'esame dei sillabi è consistito nell'identificazione di tre componenti presenti nei sillabi: (1) 'varietà regionali', (2) 'registri' e (3) 'varietà diastratiche e quelle professionali'. Nonostante che i registri e le varietà professionali appartengano alla dimensione diafasica, si è optato per un'altra modalità di classificazione, dato che di frequente le varietà diastratiche dipendono proprio dalla dimensione diafasica, costituendo un continuum senza confini precisi. L'asse diafasico indica da un lato i registri, dall'altro le lingue speciali, entrambi correlati con il contesto situazionale. La categoria delle lingue speciali si compone invece dai linguaggi altamente specializzati e quelli dal carattere professionale, ma di stampo informale o gergale (dimensione diastratica) (Berruto 1993, Sobrero 1993). Al fine di appianare le difficoltà nomenclatorie, in questo studio si è propenso per un termine generale, come 'varietà professionali' analizzate accanto alle 'varietà diastratiche', mentre i 'registri' sono stati analizzati separatamente. Ci siamo posti come secondo obiettivo quello di esaminare il corpus in chiave contrastiva, paragonando i risultati concernenti da un lato i sillabi dei lettorati e dei corsi PNJW, dall'altro i documenti classificati secondo il criterio del livello di competenza: A2/B1 e B2/C1.

In quanto alla presenza delle nozioni di varietà in tutto il corpus, i loro valori percentuali si sono distribuiti nel seguente modo: 'varietà regionali' sono apparse nel 13\% dei sillabi, 'registri' nel 44\% e 'varietà professionali/diastratiche nel $48 \%$ dei documenti.

Mettendo a confronto i documenti PNJW e quelli dei lettorati, si osservano le seguenti differenze: nella prima categoria dei programmi le nozioni relative a 'varietà regionali' compaiono nel 18\% dei sillabi, 'registri' nel $48 \%$ e 'varietà diastratiche/professionali' nel 54\%, mentre nella categoria dei lettorati i valori sono ammontati ai soli 5\% nel caso delle 'varietà regionali', e al 38\% nel caso delle altre due classi di varietà.

In riferimento al livello di competenza, le nozioni di ‘varietà regionali' si sono rilevate nel $9 \%$ dei sillabi dedicati agli studenti intermedi e nel 18\% di quelli più avanzati. La stessa relazione si nota anche nei valori inerenti ai 'registri': il 38\% versus il 52\%, nonché alle 'varietà diastratiche/professionali' : il 38\% in confronto al $61 \%$.

Dall'esame dei dati si può evincere che l'area sociolinguistica più trascurata nei sillabi sono 'varietà regionali' che compaiono nel $13 \%$ dei documenti di 
tutto il corpus e soltanto nel 5\% dei lettorati. Tale situazione dimostra che nella programmazione del contenuto dei corsi innanzi tutto di quelli di stampo generale dedicati a tutti gli studenti universitari si attribuisce una scarsa importanza alla variazione diatopica che, a sua volta, costituisce una delle caratteristiche fondamentali della realtà italiana. Le varietà diafasiche e diastratiche hanno raggiunto valori più alti, tuttavia, le relative nozioni sono apparse quasi in un terzo dei sillabi dei lettorati, il che pare insufficiente al livello accademico.

Nella tabella numero 2 sono stati illustrati i valori percentuali riguardanti la presenza nei sillabi delle nozioni appartenenti alle suddette categorie di ricerca.

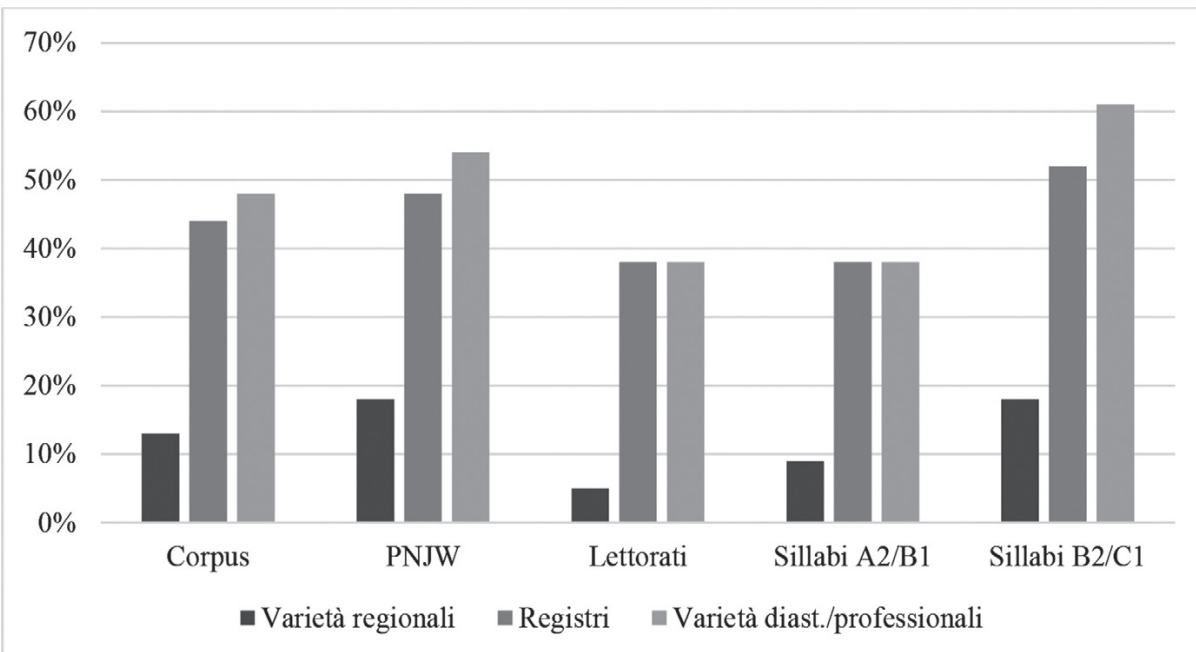

Tab. 2. Varietà di lingua presenti nei sillabi: un quadro generale

Nell'ultimo punto dell'analisi si è indagata la forma delle stesse nozioni comparse nel corpus. Nel caso della categoria 'varietà regionali' la più frequente voce presente nel $10 \%$ dei sillabi è stata la voce 'dialetti', mentre la nozione 'varietà regionali' è apparsa esclusivamente nel $3 \%$ dei casi e, inoltre, in un documento (1\%) è stata usata l'espressione 'varietà settentrionali e quelle meridionali'. Una situazione migliore concerne le nozioni relative al grado di formalità tra cui si sono registrate le seguenti nozioni: 'registri' - il 24\%, 'italiano standard' - il 21\%, 'lingua colloquiale' - il 5\%, 'volgarismi' - il 5\%, 'grado di formalità'. Nell'ambito dell'ultima tipologia di variazione in vetta alla classifica si sono collocati 'linguaggi specialistici' presenti nel $28 \%$ dei sillabi, successivamente si osserva un brusco calo dei valori numerici e, al secondo posto si è piazzata una nozione poco trasparente 'varietà stilistiche' comparendo nell' $8 \%$ dei casi, al terzo posto 'linguaggio giovanile' con il 7\%. Infine, nel 3\% dei sillabi vi è spuntata anche un'espressione difficilmente classificabile come 'fenomeni dell'italiano contemporaneo' e le singole nozioni, come: 'varietà sociali', 'linguaggio commerciale', 'linguaggio della stampa', 'linguaggio tecnico', 'linguaggio burocratico', 'gerghi professionali' e 'slang'. I più rilevanti risultati del nostro esame terminologico sono stati ricapitolati nella tabella numero 3 che, per limiti di spazio, è stata limitata soltanto ai dati inerenti a tutto il corpus dei sillabi. 


\begin{tabular}{|l|l|l|l|l|l|l|}
\hline No. & Varietà regionali & $\%$ & Registri & $\mathbf{\%}$ & $\begin{array}{l}\text { Varietà diastratiche/ } \\
\text { professionali }\end{array}$ & $\%$ \\
\hline 1. & Dialetti & $10 \%$ & Registri & $24 \%$ & Linguaggi specialistici & $28 \%$ \\
\hline 2. & Varietà regionali & $3 \%$ & Italiano standard & $21 \%$ & Varietà stilistiche & $8 \%$ \\
\hline 3. & $\begin{array}{l}\text { Dialetti settentrionali } \\
\text { e quelli meridionali }\end{array}$ & $1 \%$ & Lingua colloquiale & $5 \%$ & Linguaggio giovanile & $7 \%$ \\
\hline 4. & & & Volgarismi & $5 \%$ & Varietà sociali & $3 \%$ \\
\hline 5. & & & $\begin{array}{l}\text { Grado di formalità } \\
\text { Fenomeni dell'italiano } \\
\text { contemporaneo }\end{array}$ & $3 \%$ & $\begin{array}{l}\text { Linguaggio commerciale } \\
\text { Linguaggio della stampa } \\
\text { Ling. tecnico/burocratico } \\
\text { Gerghi professionali }\end{array}$ & $2 \%$ \\
\hline
\end{tabular}

Tab. 3. La lista delle nozioni estratte dal corpus dei sillabi

Va sottolineato ancora che al livello delle due categorie traversali dei documenti del corpus, non si notano maggiori differenze rispetto all'utilizzo delle suddette nozioni. I dati relativi a tutto il corpus corrispondono ai dati dei documenti PNJW/lettorati, nonché dei sillabi classificati secondo il criterio della competenza.

\section{Conclusioni}

Come si è potuto constatare, il contenuto dei sillabi risulta modestamente impregnato di varietà della lingua italiana, innanzi tutto nel campo della variazione regionale, che, vale la pena sottolinearlo, costituisce un tratto essenziale della realtà italiana. La situazione risulta migliore per i registri e le altre varietà diafasiche, vi si nota anche un'adeguata gradazione dei contenuti: le rispettive nozioni risultano maggiormente utilizzate nei sillabi al livello B2/C1. Si ignora invece la dimensione diastratica nel senso stretto: il 'linguaggio giovanile' compare nel 7\% dei casi e il numero di occorrenze di altre varietà del genere si rivela molto basso.

In quanto alle stesse voci presenti nei sillabi, quelle con il maggior numero di occorrenze paiono abbastanza generali e vaghe, mentre la presenza delle voci dal significato specifico è piuttosto occasionale. Mancano le nozioni che illustrano più dettagliatamente la realtà della lingua italiana nell'ambito della dimensione diafasica, ad esempio, nonostante che la voce 'registro' sia apparsa nel $24 \%$ dei casi di tutto il corpus, nei sillabi non vengono specificati i suoi particolari generi, come il registro aulico, il registro formale/informale, il registro medio, ecc. La stessa situazione concerne 'linguaggi specialistici', particolarmente importanti per gli studenti universitari, che risultano presenti solo nel $28 \%$ dei sillabi e vengono seguiti dalla nomenclatura più dettagliata solo in singoli documenti.

A questo punto è opportuno porsi la domanda se i contenuti dei sillabi dei corsi dell'italiano nelle università consentono agli studenti di conoscere appropriatamente la lingua italiana e la sua realtà sociolinguistica. L'analisi ha 
dimostrato certe lacune nella pianificazione didattica la cui qualità potrebbe essere migliorata con l'inclusione degli elementi che rispondano meglio ai bisogni di questo gruppo degli apprendenti.

\section{Bibliografia}

Beacco, Jean Claude, Byram Michael (2007). From linguistic diversity to plurilingual education: Guide for the development of language education Policies in Europe. Main Version, Strasbourg: Council of Europe.

Berruto, Gaetano (1993). Varietà diamesiche, diastratiche, diafasiche, in: Introduzione all'italiano contemporaneo. La variazione e gli usi [a cura di Alberto A. Sobrero], Roma-Bari: Laterza, pp. 37-92.

Cini, Lucia (2011). La programmazione per la L2, in: Insegnare italiano a stranieri [a cura di Pierangela Diadori], Milano: Le Monnier, pp. 145-156.

Cisowska, Galina (2012). Kształcenie językowe na studiach niefilologicznych, Radom: Wydawnictwo Politechniki Radomskiej.

Communiqué of the Conference of Ministers responsible for Higher Education (2003). Realising the European Higher Education Area, Berlin: http://www.ehea. info/media.ehea.info/file/2003_Berlin/28/4/2003_Berlin_Communique_English_577284.pdf.

Consiglio d'Europa (2002). Quadro comune europeo di riferimento per le lingue: apprendimento, insegnamento, valutazione (QCER), La Nuova Italia-Oxford, Firenze.

ELC (2001). Multilingualism and new learning environments. Workshop 1: Universities and language policy in Europe. Reference Document, Berlin: Freie Universität.

Gajewska, Elżbieta (2008). Kształcenie nauczycieli języków specjalistycznych, in: Nauczanie języków obcych w szkole wyższej [a cura di Anna M. Harbig], Białystok: Wydawnictwo Uniwersytetu w Białymstoku, pp. 75-84.

Główny Urząd Statystyczny (GUS) (2017). Szkoły wyższe i ich finanse w 2016 r., Warszawa: Zakład Wydawnictw Statystycznych.

Kaliska, Marta (2018). Model uczenia języków obcych w szkole wyższej na przykładzie jezyka włoskiego. Założenia teoretyczne, metodologia nauczania i zintegrowany rozwój kompetencji, Warszawa: UW IKSI.

Kraśniewski, Andrzej (2009). Proces Boloński. To już 10 lat, Warszawa: FRSE.

Mezzadri, Marco (2016). Le politiche linguistiche europee: tra continuità e cambiamento, in: Le lingue in Italia, le lingue in Europa: dove siamo, dove andiamo [a cura di Carlos A. Melero Rodríguez], Venezia: Edizioni Ca' Foscari, pp. 11-20.

Sobrero, Alberto (1993). Lingue speciali, in: Introduzione all'italiano contemporaneo. La variazione e gli usi [a cura di Alberto A. Sobrero], Roma-Bari: Laterza, pp. 237-277. 


\section{Language varieties in the Italian Language Teaching Syllabi at Polish Universities}

The article presents the findings of the case study on Italian courses' syllabi in terms of language varieties present in the university syllabi. The corpus consists of 100 syllabi collected from the 10 biggest Polish universities. Their content has been analysed in order to identify the variety areas which are mostly emphasized or completely omitted in such teaching programmes.

Keywords: higher education, syllabus, curriculum, Italian language teaching, language varieties 
